\title{
Study of the Changes of Gastric Wall Mucosa Optical Properties under the Impact of Aqueous Solutions of Haemoglobin and Glucose for Improving Conditions of the Laser Coagulation
}

\author{
Alexey N. Bashkatov ${ }^{1,2}$, Elina A. Genina ${ }^{1,2 *}$, Veniamin A. Grishaev ${ }^{3}$, Sergey V. Kapralov ${ }^{4}$, \\ Vyacheslav I. Kochubey ${ }^{1,2}$, and Valery V. Tuchin ${ }^{1,2,5}$ \\ ${ }^{1}$ Saratov State University, 83 Astrakhanskaya Str., Saratov 410012, Russia \\ ${ }^{2}$ National Research Tomsk State University, 36 Lenin Ave., Tomsk 634050, Russia \\ ${ }^{3}$ Saratov City Clinical Hospital No. 6 named after Acad. V.N. Koshelev, 15 Gvardeyskaya Str., Saratov 410033, Russia \\ ${ }^{4}$ Engels City Clinical Hospital No. 1, 6 Vesennaya Str., Engels, Saratov Region 413116, Russia \\ ${ }^{5}$ Laboratory of Laser Diagnostics of Technical and Living Systems, Institute of Precision Mechanics and Control, \\ Russian Academy of Sciences, 24 Rabochaya Str., Saratov 410028, Russia
}

* e-mail: eagenina@yandex.ru

\begin{abstract}
The paper presents the results of ex vivo studies of the possibility to control the absorption and scattering properties of the human gastric wall mucosa. For the first time we obtained the increase of the tissue absorption coefficient in the range 350-1250 $\mathrm{nm}$ by 2-4.5 times under the injection of haemoglobin solution with the concentration of $70 \mathrm{~g} / \mathrm{L}$ into the mucosa. The observed increase of the absorbed energy fraction by nearly $65-90 \%$ was accompanied by almost $50-60 \%$ decrease of the penetration depth at the wavelengths of the sources widely used for laser ablation and coagulation of gastric mucosa paraplasms. Under the injection of $40 \%$ glucose solution into the mucosa, we observed the reduction of the absorption coefficient in the spectral region of water absorption bands approximately by $20 \%$ and the reduction of the transport scattering coefficient by nearly $24-27 \%$ in the spectral range $350-2500 \mathrm{~nm}$. Increasing of the depth of laser radiation penetration in this case amounted to $15-17 \%$ in the range $800-1100$ $\mathrm{nm}$. The performed studies show the possibility in principle to control the optical parameters of the gastric wall tissues from the point of view of both varying the laser radiation penetration depth and regulating the tissue absorbance and, hence, the power of laser radiation, which, in turn, will allow more safety and less invasion in the course of laser therapeutic procedures. (C) 2017 Journal of Biomedical Photonics \& Engineering.
\end{abstract}

Keywords: Mucose; spectroscopy; optical properties control.

Paper \#3272 received 6 Dec 2017; revised manuscript received 18 Dec 2017; accepted for publication 19 Dec 2017; published online 31 Dec 2017. doi: 10.18287/JBPE17.03.040304. [Saratov Fall Meeting 2017 Special Issue].

\section{References}

1. C. T. Germer, D. Albrecht, C. Isbert, J. Ritz, A. Roggan, and H. J. Buhr, "Diffusing fibre tip for minimally invasive treatment of liver tumours by interstitial laser coagulation (ILC): an experimental ex vivo study," Lasers in Medical Science 14, 32-39 (1999).

2. R. Sroka, C. G. Schmedt, S. Steckmeier, O. A. Meissner, W. Beyer, G. Babaryka, and B. Steckmeier, "Ex-vivo investigation of endoluminal vein treatment by means of radiofrequency and laser irradiation," Medical Laser Application 21, 15-22 (2006). 
3. C. M. Cilip, A. E. Ross, J. P. Jarow, and N. M. Fried, "Application of an optical clearing agent during noninvasive laser coagulation of the canine vas deferens," Journal of Biomedical Optics 15(4), 048001 (2010).

4. G. R. Schweinsberger, C. M. Cilip, S. R. Trammell, H. Cherukuri, and N. M. Fried, "Noninvasive laser coagulation of the human vas deferens: optical and thermal simulations," Lasers in Surgery and Medicine 43, 443-449 (2011).

5. J. T. Au, A. Mittra, J. Wong, S. Carpenter, J. Carson, D. Haddad, S. Monette, P. Ezell, S. Patel, and Y. Fong, "Flexible $\mathrm{CO}_{2}$ laser and submucosal gel injection for safe endoluminal resection in the intestines," Surgical Endoscopy 26(1), 47-52 (2012).

6. S. Kim, M. T. Hossain, D. H. Lee, and J. K. Kim, “Analysis of opto-thermal interaction of porcine stomach tissue with 808-nm laser for endoscopic submucosal dissection," Journal of Innovative Optical Health Sciences $8(6), 1550043$ (2015)

7. V. Rubtsov, Yu. Chalyk, and A. Bashkatov, "Rational choice of laser wavelength for endoscopic photodestruction of colorectal polyps," Vrach 12, 83-85 (2013) [in Russian].

8. J.-H. Cho, J. Y. Cho, M.-Y. Kim, S. R. Jeon, T. H. Lee, H. G. Kim, S. Y. Jin, and S. J. Hong, "Endoscopic submucosal dissection using a thulium laser: Preliminary results of a new method for treatment of gastric epithelial neoplasia," Endoscopy 45(9), 725-728 (2013).

9. V. V. Tuchin, "Tissue optics and photonics: light-tissue interaction," Journal of Biomedical Photonics \& Engineering 1(2), 98-134 (2015).

10. T. Uraoka, T. Fujii, Y. Saito, T. Sumiyoshi, F. Emura, P. Bhandari, T. Matsuda, K. Fu, and D. Saito, "Effectiveness of glycerol as a submucosal injection for EMR," Gastrointestinal Endoscopy 61, 736-740 (2000).

11. H. Yamamoto, H. Kawata, K. Sunada, K. Satoh, Y. Kaneco, K. Ido, and K. Sugano, "Success rate of curative endoscopic mucosal resection with circumferential mucosal incision assisted by submucosal injection of sodium hyaluronate," Gastrointestinal Endoscopy 56, 507-512 (2002).

12. I. Kumano, M. Ishihara, S. Nakamura, S. Kishimoto, M. Fujita, H. Hattori, T. Horio, Y. Tanaka, K. Hase, and T. Maehara, "Endoscopic submucosal dissection for pig esophagus by using photocrosslinkable chitosan hydrogel as submucosal fluid cushion," Gastrointestinal Endoscopy 75(4), 841-848 (2012).

13. A. N. Bashkatov, E. A. Genina, V. I. Kochubey, A. A. Gavrilova, S. V. Kapralov, V. A. Grishaev, and V. V. Tuchin, "Optical properties of human stomach mucosa in the spectral range from 400 to 2000 nm: prognosis for gastroenterology," Medical Laser Application 22, 95-104 (2007).

14. K. M. Giraev, N. A. Ashurbekov, and O. V. Kobsev, "Optical spectra of some pathological conditions of stomach tissues," International Journal of Modern Physics B 20(1), 25-36 (2006).

15. K. M. Giraev, N. A. Ashurbekov, and M. A. Lakhina, "Optical absorption and scattering spectra of pathological stomach tissues," Journal of Applied Spectroscopy 78(1), 95-102 (2011).

16. H. J. Wei, D. Xing, B. H. He, H. M. Gu, G. Y. Wu, and X. M. Chen, "Using of oblique incident laser beam to measure the optical properties of stomach mucosa/submucosa tissue", BMC Gastroenterology 9, 64, (2009)

17. R. K. Wang, X. Xu, Y. He, and J. B. Elder, "Investigation of optical clearing of gastric tissue immersed with hyperosmotic agents," IEEE Journal of Selected Topics in Quantum Electronics 9(2), 234-242 (2003).

18. X. Xu, R. Wang, and J. B. Elder, "Optical clearing effect on gastric tissues immersed with biocompatible chemical agents investigated by near infrared reflectance spectroscopy," Journal of Physics D: Applied Physics $36,1707-1713$ (2003).

19. Y. He, and R. K. Wang, "Dynamic optical clearing effect of tissue impregnated with hyperosmotic agents and studied with optical coherence tomography," Journal of Biomedical Optics 9(1), 200-206 (2004).

20. X. Xu, and R. K. Wang, "Synergistic effect of hyperosmotic agents of dimethyl sulfoxide and glycerol on optical clearing of gastric tissue studied with near infrared spectroscopy," Physics in Medicine \& Biology 49, 457-468 (2004).

21. H. Xiong, Z. Guo, C. Zeng, L. Wang, Y. He, and S. Liu, “Application of hyperosmotic agent to determine gastric cancer with optical coherence tomography ex vivo in mice," Journal of Biomedical Optics 14(2), 024029 (2009).

22. V. V. Tuchin, D. M. Zhestkov, A. N. Bashkatov, and E. A. Genina, "Theoretical study of immersion optical clearing of blood in vessels at local hemolysis," Optics Express 12(13), 2966-2971 (2004).

23. S. A. Prahl, M. J. C. van Gemert, and A. J. Welch, "Determining the optical properties of turbid media by using the adding-doubling method," Applied Optics 32(4), 559-568 (1993).

24. A. N. Bashkatov, E. A. Genina, V. I. Kochubey, V. S. Rubtsov, E. A. Kolesnikova, and V. V. Tuchin, "Optical properties of human colon tissue in the $350-2500 \mathrm{~nm}$ spectral range," Quantum Electronics 44(8), 779-784 (2014).

25. A. N. Bashkatov, E. A. Genina, M. D. Kozintseva, V. I. Kochubei, S. Yu. Gorodkov, and V. V. Tuchin, "Optical properties of peritoneal biological tissues in the spectral range of 350-2500 nm," Optics and Spectroscopy 120(1), 1-8 (2016). 
26. L. Wang, S. L. Jacques, and L. Zheng, "MCML - Monte Carlo modeling of light transport in multi-layered tissues," Computer Methods and Programs in Biomedicine 47, 131-146 (1995).

27. W.H. Press, S. A. Tuekolsky, W. T. Vettering, and B. P. Flannery, "Numerical recipes in C: The art of scientific computing," Cambridge University Press, Cambridge, New York (1992).

28. V. V. Tuchin, "Tissue optics: Light Scattering Methods and Instruments for Medical Diagnosis. Third edition," SPIE Press, Washington, Bellingham (2015).

\section{Introduction}

The contactless laser coagulation and ablation of pathologic formations in different organs is widely used in modern clinics [1-8]. The minor invasiveness of the procedure reduces the risk of postoperative complications. The sources used for this purpose include, particularly, the ytterbium $(1075 \mathrm{~nm})$ [3], $\mathrm{CO}_{2}$ (9.4-10.6 $\mu \mathrm{m})$ [5], Nd:YAG $(1064 \mathrm{~nm})[1,4]$, diode $(980,808,810 \mathrm{~nm})[2,6,7]$, and other lasers. The main advantage of lasers generating at the wavelengths that coincide with the absorption bands of water is the small depth of light penetration into tissues, which prevents the damage of the adjacent healthy tissue, underlying the affected area $[5,8]$. However, in recent time the cheaper diode lasers become more and more widely used.

The radiation from infrared diode lasers in the range 800-1100 nm penetrates deep enough, since in this range the absorption of such tissue components as haemoglobin, melanin, proteins, and water is relatively small [9]. Thus, the danger of tissue damage or perforation arises. To prevent these complications, the authors of some papers propose to increase the thickness of the irradiated object. Thus, e.g., according to the data of Refs. [10-12], in order to protect the mucosa of stomach and intestinal wall in the course of laser endoscopic resection, the layers of mucosa and submucosa were separated by a certain spacing, filled with glycerol, sodium hyaluronate, or hydrogel.

An alternative possible approach is to optimise the laser impact by varying the optical parameters of the tissue. The control of optical parameters can be implemented both by enhancing the absorption properties of the object itself and by reducing the scattering in the tissues adjacent to the lesion focus. In the first case, on the one hand, the laser beam penetration depth is reduced and, on the other hand, the fraction of energy absorbed in the lesion focus is essentially increased, thus improving the laser coagulation efficiency. In the second case, the precision of laser radiation focusing is facilitated.

The absorption and scattering properties of the stomach wall mucosa are well-studied [13-16]. It has been shown that the use of biocompatible immersion agents, such as solutions of glycerol, propylene glycol, etc., results in efficient optical clearing (i.e., light scattering reduction) of the gastric tissues in the near IR spectral region [17-21]. Earlier we proposed to use haemoglobin in order to control the scattering properties of blood by creating a local haemolysis site in a blood vessel [22]. In the present paper, we propose to use the blood haemoglobin as an absorbing agent.

The aim of the paper is to study the effect of aqueous haemoglobin solution and aqueous 40\% glucose solution on the optical properties of gastric wall mucosa in order to improve the conditions for laser coagulation in the visible and near IR spectral ranges.

\section{Materials and methods}

The study has been performed with 15 samples of human gastric wall mucosa from different patients, obtained in the course of planned operations or anatomic investigations. The experimental studies were approved by the Ethics Committee of V.I. Razumovsky Saratov State Medical University. Immediately after the autopsy, the samples were placed in $0.9 \%$ solution of $\mathrm{NaCl}$ and were kept in it until the conduction of spectral measurements during 4-8 hours at the temperature $\sim 4{ }^{\circ} \mathrm{C}$.

Immediately before the measurements, the samples having the dimensions of nearly $15 \times 20 \mathrm{~mm}^{2}$ were cut from the tissue. Five samples were used as a control group. They were not subjected to the impact of any agents. The next five samples were subjected to multiple injections of the standard aqueous solution of haemoglobin (OOO "AGAT-MED", Russia) with the concentration $70 \mathrm{~g} / \mathrm{L}$. The total volume of the solution injected into each sample amounted to $\sim 0.1 \mathrm{~mL}$. Into the rest five samples the standard $40 \%$ aqueous solution of glucose (NPO "Microgen", Russia) was injected in a similar way. Multiple injection of preparation microdoses over the mucosa area before the laser exposure was intended to model the conditions of the endoscopic laser coagulation procedure. Then the samples were placed between two microscope slides without compression. The edges of the slides were fixed. The sample thickness was measured by means of a micrometer at five points. The accuracy of the measurement was $\pm 10 \mu \mathrm{m}$. The obtained values were averaged. The resulting thickness of the samples varied from $1 \mathrm{~mm}$ to $2.5 \mathrm{~mm}$.

The coefficients of total transmission and diffuse reflection were measured not later than in 10-15 minutes after the injections. The optical properties of the tissue were studied in the spectral range $350-2500 \mathrm{~nm}$ using the spectrophotometer LAMBDA 950 (PerkinElmer Ltd, UK) with integrating sphere, which is a doublechannel monochromator with the built-in system of control and signal recording. The photometric accuracy of measurements amounted to $\pm 0.0006 \mathrm{Abs}$. As a source of radiation, we used a halogen incandescent lamp. The 
spot dimensions of the light beam incident on the sample were $5 \times 5 \mathrm{~mm}^{2}$. The scanning velocity was 2 $\mathrm{nm} / \mathrm{s}$.

To process the experimental results and to determine the optical parameters of mucosa we used a combined method. At the first stage of the method, the measurement data were processed using the inverse adding-doubling (IAD) method [23]. Then the accuracy of the obtained values of the absorption coefficient $\left(\mu_{\mathrm{a}}\right)$ and transport scattering coefficient $\left(\mu_{\mathrm{s}}^{\prime}\right)$ was improved using the inverse Monte Carlo method [24, 25] by minimising the target function

$$
F\left(\mu_{\mathrm{a}}, \mu_{\mathrm{s}}^{\prime}\right)=\left(R_{\mathrm{d}}^{\exp }-R_{\mathrm{d}}^{\text {calc }}\left(\mu_{\mathrm{a}}, \mu_{\mathrm{s}}^{\prime}\right)\right)^{2}+\left(T_{\mathrm{t}}^{\exp }-T_{\mathrm{t}}^{\text {calc }}\left(\mu_{\mathrm{a}}, \mu_{\mathrm{s}}^{\prime}\right)\right)^{2} \text {. }
$$

Here $R_{\mathrm{d}}^{\text {exp }}, T_{\mathrm{t}}^{\text {exp }}, R_{\mathrm{d}}^{\text {calc }}, T_{\mathrm{t}}^{\text {calc }}$ are the values of the diffuse reflection coefficient $\left(R_{\mathrm{d}}\right)$ and total transmission coefficient $\left(T_{\mathrm{t}}\right)$ experimentally measured and theoretically calculated using the Monte Carlo method [26], with the geometry of the studied medium and the experiment taken into account. As an iteration procedure, we used the Nelder-Mead simplex-method thoroughly described in Ref. [27]. The iteration procedure was continued until the agreement of the measured and the calculated data to a given accuracy $(<0.1 \%)$.

The light penetration depth is one of the most important characteristics for the correct determination of irradiation doze in photochemical and photodynamic therapy of different diseases, as well as the optical radiation dosimetry in the laser surgery of gastric ulcer. The depth of radiation penetration into the tissue $(\delta)$ was evaluated using the relation $\delta=1 / \sqrt{3 \mu_{\mathrm{a}}\left(\mu_{\mathrm{a}}+\mu_{\mathrm{s}}^{\prime}\right)}$ [28].

\section{Results and discussion}

Figures 1 and 2 present the spectra of the absorption coefficient $\left(\mu_{\mathrm{a}}\right)$ and the transport scattering coefficient $\left(\mu_{\mathrm{s}}^{\prime}\right)$ of the gastric wall mucosa before and after the injection of aqueous haemoglobin solution. The averaging was executed over five samples of the tissue.

From Fig. 1 it follows that in the spectral range from 350 to $1250 \mathrm{~nm}$ one can observe an essential increase of the absorption coefficient of the mucosa (by 2-4.5 times depending on the wavelength). In the spectral range $1250-2500 \mathrm{~nm}$ the increase is expressed essentially weaker. Such behaviour of the absorption coefficient is related to the characteristic absorption of haemoglobin in the visible wavelength range. From Fig. 2 it follows that the injection of the aqueous solution of haemoglobin has practically no effect on the scattering characteristics of the mucosa.

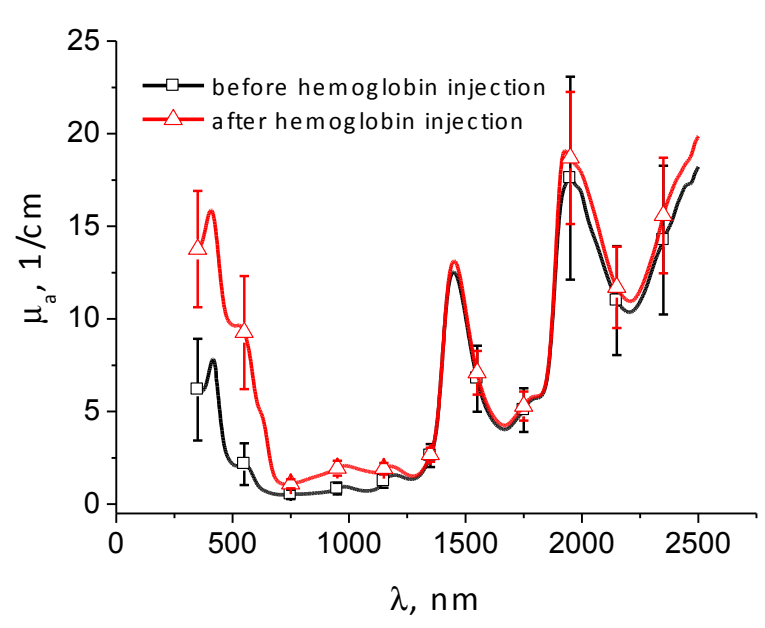

Fig. 1 Absorption spectrum of the human gastric wall mucosa measured before and after the injection of aqueous haemoglobin solution. Vertical bars show the root-mean-square deviation.

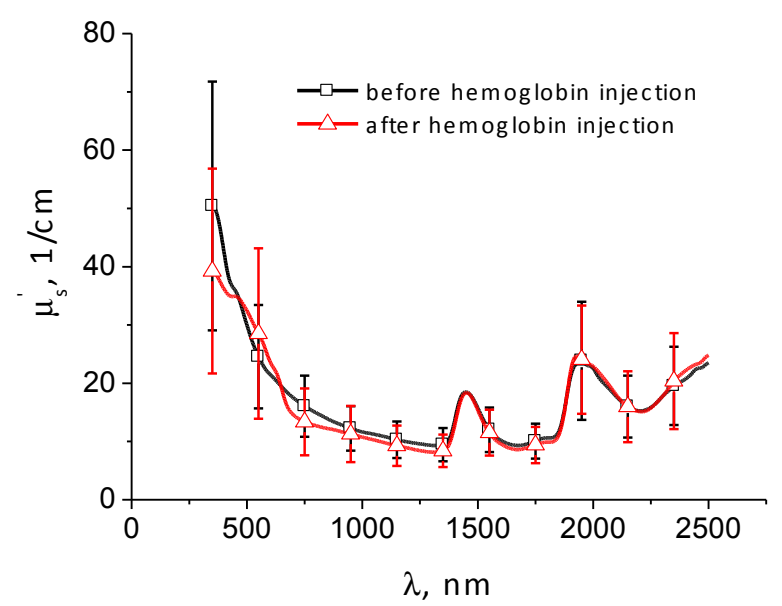

Fig. 2 Spectrum of transport scattering coefficient of human gastric wall mucosa measured before and after the injection of aqueous haemoglobin solution. Vertical bars show the root-mean-square deviation.

The result of estimating the depth $(\delta)$ of light penetration into the tissue is shown in Fig. 3. The used expression is applicable to the case when the tissue surface is uniformly illuminated by the radiation from a point source, located at some distance from the surface. This corresponds to the conditions of real laser surgery of gastric wall, since in this case the illuminating probe is introduced directly into the stomach cavity without a contact with the mucosa surface. The depth of light penetration into the mucosa was calculated using the values of the absorption coefficient (see Fig. 1) and the transport scattering coefficient (see Fig. 2).

Another important parameter is the fraction of the incident radiation energy absorbed in the mucosa. This parameter was calculated in the course of Monte Carlo modelling, and the result is presented in Fig. 3, too.

In this figure one can clearly see that the depth of laser radiation penetration essentially decreases in the 
visible range of wavelengths (at the wavelength of $\mathrm{Nd}$ :YAG laser radiation $(1064 \mathrm{~nm})$ the penetration depth decreases by nearly $60 \%$, whereas the fraction of absorbed energy at this wavelength increases by nearly $90 \%$ ). The computer simulation allows the evaluation of the change of the absorbed energy fraction at the wavelengths of diode-based laser systems used for the photodestruction of tissue neoplasms. Thus, for the wavelength $810 \mathrm{~nm}$ the injection of haemoglobin aqueous solution leads to the increase of the absorbed energy fraction by nearly $80 \%$ with almost $50 \%$ reduction of the laser radiation penetration depth. For the wavelength $970 \mathrm{~nm}$, the increase of the absorbed energy fraction amounts to nearly $65 \%$ with about $50 \%$ decrease of the laser radiation penetration depth.

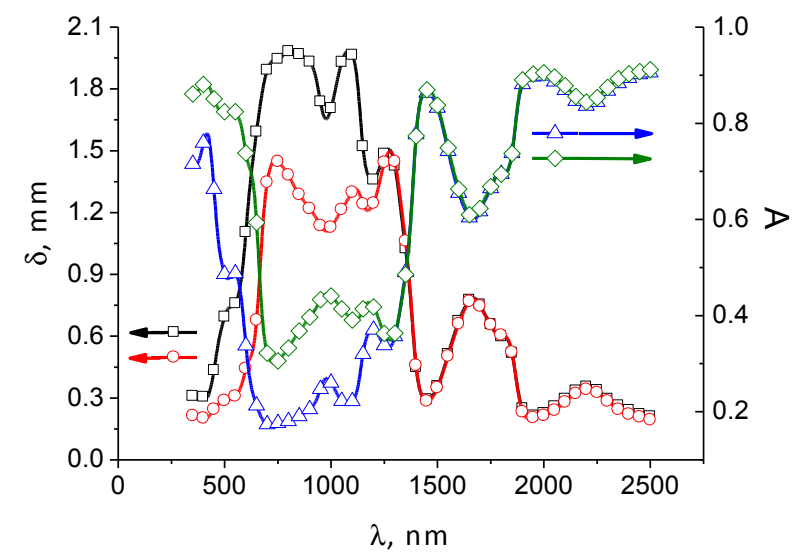

Fig. 3 Wavelength dependence of the depth of radiation penetration into the human gastric wall mucosa $(\delta)$ (black line, $\square-$ before the haemoglobin injection; red line, $\bigcirc$ - after the haemoglobin injection), calculated from the experimental data of Figs. 1 and 2. Results of Monte Carlo simulation of the fraction of photons absorbed in the mucosa (A) versus the wavelength (blue line, $\Delta$ - before the haemoglobin injection; green line, $\diamond$ - after the haemoglobin injection).

Figures 4 and 5 present the spectra of absorption and transport scattering coefficients of the gastric wall mucosa before and after the injection of aqueous $40 \%$ glucose solution. The averaging was performed over five samples of tissue.

In Fig. 4, one can clearly see that the injection of glucose solution into the mucosa practically does not change its absorption properties, except the region of water absorption bands in the near IR spectral region with the peaks at 1450 and $1940 \mathrm{~nm}$. This fact is due to the replacement of the mucosa interstitial fluid with the glucose solution and, hence, the decrease of water content in the tissue.

Figure 5 clearly demonstrates that the glucose injection leads to the essential $(\sim 20 \%)$ reduction of the transport scattering coefficient all over the range of wavelengths under study (24-27\%), which leads to the increase of the laser radiation penetration depth (see Fig. 6).
The computer simulation shows that the growth of the penetration depth of laser radiation is about $15 \%$ for the wavelength $810 \mathrm{~nm}$, about $17 \%$ for $970 \mathrm{~nm}$, and about $16 \%$ for $1064 \mathrm{~nm}$. Practically no increase of the fraction of photons absorbed in the mucosa is observed (see Fig. 6).

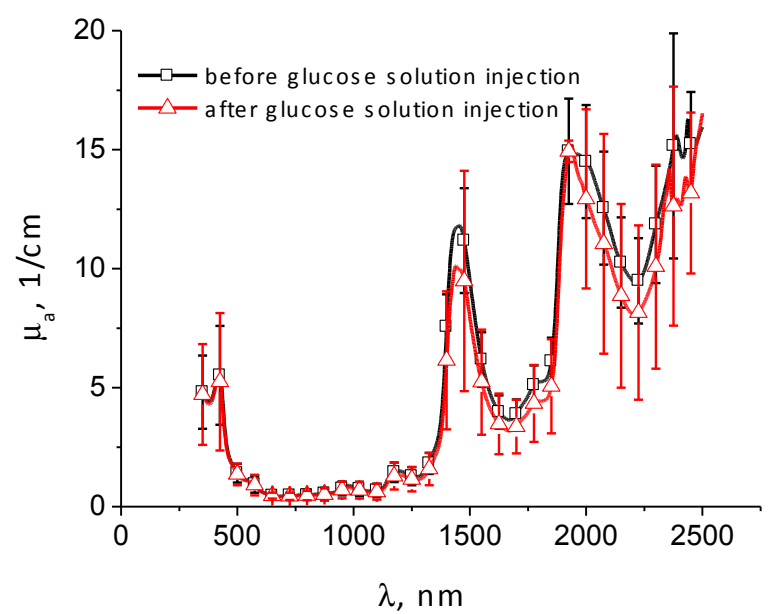

Fig. 4 Absorption spectrum of the human gastric wall mucosa, measured before and after the injection of aqueous $40 \%$ glucose solution. Vertical bars show the root-mean-square deviation.

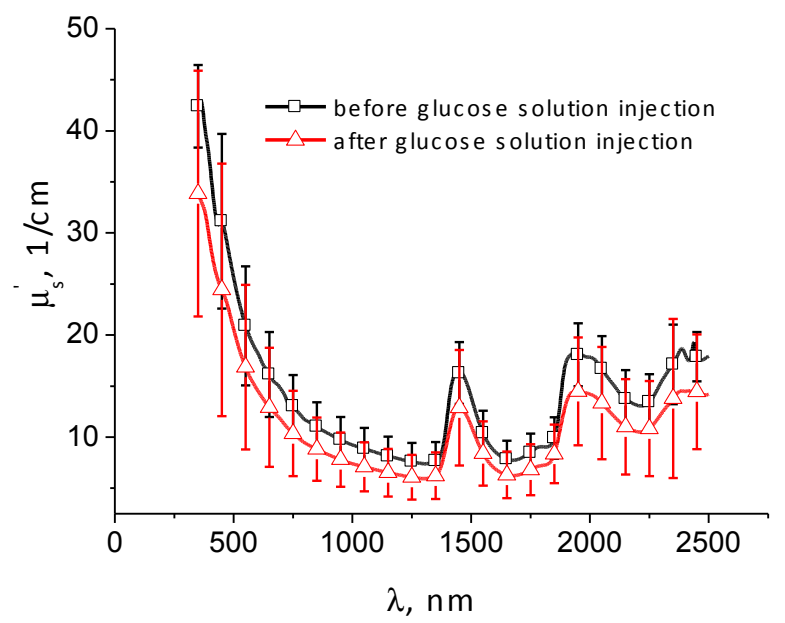

Fig. 5 Spectrum of transport scattering coefficient of the human gastric wall mucosa measured before and after the injection of aqueous $40 \%$ glucose solution. Vertical bars show the root-mean-square deviation.

The obtained results of the mucosa optical clearing agree well with the data of Refs. [17-21]. Thus, in Refs. $[17,18,20]$ it is shown that the absorption coefficient of a fresh sample of porcine gastric mucosa at the wavelengths 1450 and $1936 \mathrm{~nm}$ decreases under the action of glycerol and dimethyl sulphoxide (DMSO), which is an evidence of water desorption caused by the applied agents. The authors observed the increase of the optical coherence tomography (OCT) probing depth at the wavelength $1300 \mathrm{~nm}$ using the glycerol and DMSO solutions as immersion clearing agents $[17,19]$. The authors of Ref. [21] also obtained increased transparency of the murine gastric wall mucosa under 
the application of $20 \%$ glucose solution in saline at the wavelength $830 \mathrm{~nm}$ in OCT studies.

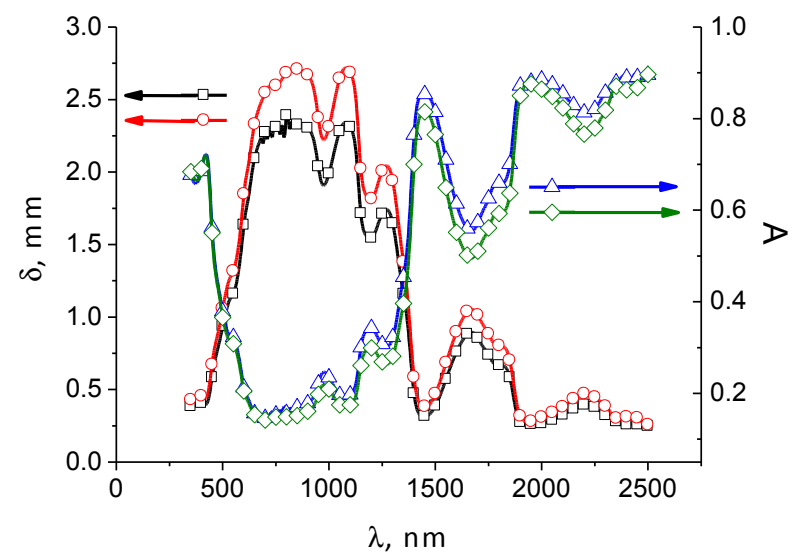

Fig. 6 Wavelength dependence of the depth of radiation penetration into the human gastric wall mucosa $(\delta)$ (black line, $\square-$ before the glucose injection; red line, $\circ$ - after the glucose injection), calculated from the experimental data of Figs. 4 and 5. The result of Monte Carlo simulation of the fraction of photons absorbed in the mucosa (A) versus the wavelength (blue line, $\Delta$ - before the glucose injection; green line, $\diamond$ - after the glucose injection).

\section{Conclusion}

As a result of the performed studies of the possibility to control the absorption and scattering properties of the gastric wall mucosa, we report the first experimental observation of the tissue absorption coefficient increase in the range 350-1250 due to the injection of haemoglobin solution into the tissue.

After the injection of $40 \%$ glucose solution into the mucosa, we observed a decrease of the absorption coefficient in the region of water absorption bands and the decrease of transport scattering coefficient all over the range under study $(350-2500 \mathrm{~nm})$.

The performed studies show the possibility to control the optical properties of the gastric wall tissues from the point of view of both changing the depth of laser radiation penetration and regulating the tissue absorbance and, therefore, the incident radiation power. Increasing the absorption in the affected tissues will allow the reduction of the incident radiation power, which, in turn, will reduce the risk of damaging healthy tissues surrounding the lesion area, which can find application in planning the operations of gastric ulcer treatment using up-to-date laser technologies.

\section{Disclosures}

All authors declare that there is no conflict of interests in this paper.

\section{Acknowledgments}

The work was carried out within the framework of the project "Photoacoustic Technologies for Early Teranostics of Metastatic Tumours" supported by the grant from the Government of the Russian Federation for the state support of research conducted under the supervision of leading scientists in Russian institutions of higher education and research organisations. 